\title{
Response of Flame Thickness and Propagation Speed under Intense Turbulence in Spatially Developing Lean Premixed Methane-Air jet Flames
}

\author{
Ramanan Sankaran \\ Oak Ridge National Laboratory, Oak Ridge, TN 37831-6008, USA \\ Evatt R. Hawkes \\ The University of New South Wales, Sydney, NSW 2052, Australia \\ Chun S. Yoo \\ Ulsan National Institute of Science and Technology, Ulsan 689-798, Republic of Korea \\ Jacqueline H. Chen \\ Sandia National Laboratories, Livermore, CA 94551-0969, USA
}

\begin{abstract}
Direct numerical simulations of three-dimensional spatially-developing turbulent Bunsen flames were performed at three different turbulence intensities. The simulations were performed using a reduced methane-air chemical mechanism which was specifically tailored for the lean premixed conditions simulated here. A planar-jet turbulent Bunsen flame configuration was used in which turbulent preheated methane-air mixture at 0.7 equivalence ratio issued through a central jet and was surrounded by a hot laminar coflow of burned products. The turbulence characteristics at the jet inflow were selected such that combustion occured in the thin reaction zones (TRZ) regime. At the lowest turbulence intensity, the conditions fall on the boundary between the TRZ regime and the corrugated flamelet regime, and progressively


moved further into the TRZ regime by increasing the turbulent intensity. The data from the three simulations was analyzed to understand the effect of turbulent stirring on the flame structure and thickness. Statistical analysis of the data showed that the thermal preheat layer of the flame was thickened due to the action of turbulence, but the reaction zone was not significantly affected. A global and local analysis of the burning velocity of the flame was performed to compare the different flames. Detailed statistical averages of the flame speed were also obtained to study the spatial dependence of displacement speed and its correlation to strain rate and curvature.

NOTICE: This manuscript has been authored by UT-Battelle, LLC under Contract No. DE-AC05-00OR22725 with the U.S. Department of Energy. The United States Government retains and the publisher, by accepting the article for publication, acknowledges that the United States Government retains a non-exclusive, paid-up, irrevocable, world-wide license to publish or reproduce the published form of this manuscript, or allow others to do so, for United States Government purposes. The Department of Energy will provide public access to these results of federally sponsored research in accordance with the DOE Public Access Plan (http://energy.gov/downloads/doe-public-access-plan).

Keywords: Premixed flame, turbulent combustion, direct numerical simulation, Flame speed, thin reaction zones, lean premixed, natural gas 


\section{Introduction}

In many practical applications for power generation, such as stationary gas turbines, there has been a strong interest in achieving lean premixed combustion. The advantages of operating at lean mixture conditions are 5 high thermal efficiency and low emissions of NOx due to lower flame temperatures. Lean premixed combustion tends to be different from combustion at stoichiometric conditions in certain key aspects. For example, lean flames tend to be thicker and propagate more slowly due to the lower flame temperature. A thicker flame is more susceptible to disruption and stirring by the small scale eddies of turbulence. Similarly, the turbulent velocity fluctuations can have a higher impact due to the lower flame speed. For these reasons, the influence of turbulence on premixed flame propagation is expected to be different at lean conditions compared to stoichiometric conditions.

Traditionally, the phenomenal description and modeling of turbulent premixed flames has been based on the ratio of the turbulence scales to the characteristic flame scales. In the regime where the flame time scale is shorter than the entire range of turbulence time scales, the chemistry and turbulence do not interact and hence can be decoupled. Such a flame can be represented by a locally thin and one-dimensional flamelet. However, when the flame is thicker and the relative turbulence intensity higher, as could be expected in lean premixed combustion for the reasons mentioned above, the small eddies of turbulence can enter the flame structure. Peters [1] has provided a model for flame propagation in the regime where the turbulence scales are capable of penetrating and influencing the preheat zone, but are incapable of penetrating the reaction zones. This regime is called the thin reaction zones 
(TRZ) regime.

Premixed flame structure consists of a broader preheat layer upstream of a narrower reaction layer. Usual modeling approaches for the TRZ regime assume that the turbulent eddies can enter and influence the preheat layer preheat layer, is not penetrated. The assumption of an order of magnitude disparity in the thickness of the preheat layer versus the reaction layer is based on theoretical analysis of the flame structure using activation energy asymptotics. It is customary to define the preheat layer thickness based on full width at half maximum of heat release rate. In practical fuels such as methane-air, especially at lean and preheated conditions, the preheat layer is only around two to three times wider than the reaction layer. It needs to be determined if the reaction layer indeed stays intact in the entire TRZ regime despite the fact that it is not as thin as commonly assumed.

There are several outstanding questions on the response of flames in the TRZ regime that remain to be answered definitively. First, it is not clear whether the flame dynamics in this regime are dominated by the entrainment of small eddies into the flame structure or the large scale flow straining which does not alter the flame structure. The penetration of the local flame structure by small eddies is expected to cause thickened flames in the TRZ regime [1, 2]. However the competetive effect of the large scale structure is expected to thin the flame. Thus, it unclear whether flames are thicker or thinner in the TRZ regime. Several experimental studies in this regime have 50 reported [3, 4] thicker flames, while others have reported thinner flames [5, 6]. 
A more important quantity of interest is the burning velocity of turbulent premixed flames, both from fundamental understanding and modeling perspectives. One important question [7] encountered in premixed combustion is, "how fast can we burn?". In an attempt to quantify and model the influence of turbulence on premixed combustion, a premixed flame is treated as a surface separating the fresh reactants and burnt products. This allows the influence of turbulence on combustion to be distinguished into two important phenomena - (i) an increase in the flame surface area within the same volume through wrinkling and aerodynamic straining of the flame (ii) a change in the burning rate of the flame per unit surface area with respect to a laminar flame. Based on this concept, a model for the turbulent burning velocity has been suggested by Bray [8], Candel and Poinsot [9]. In this model, the mean turbulent burning velocity can be written as,

$$
S_{\mathrm{T}}=S_{\mathrm{L}} \cdot I_{0} \cdot A^{\prime}
$$

where $S_{\mathrm{L}}$ is the laminar burning velocity, $I_{0}$ is the efficiency factor to account for change in burning velocity per unit area and $A^{\prime}$ accounts for the increase in surface area. Formulating turbulent burning velocity in this manner allows models to be derived for the two contributing factors, $I_{0}$ and $A^{\prime}$. For example, one can model the change in burning velocity per unit area of the flame, $I_{0}$, based on a laminar strained flame and then account for the increase in flame surface area through a flame surface density model [10]. An improved understanding of the relative contribution of $I_{0}$ and $A^{\prime}$ on the burning velocity in the TRZ regime is required. Also, the physical mechanisms and relative contributions of flame surface area generation and flame surface burning efficiency needs to be understood for improving premixed combustion models in 
the TRZ regime. This is made possible through the ability to perform high fidelity direct numerical simulations (DNS) of turbulent premixed flames in statistically stationary configurations that allows quantitative and converged statistics of the relevant quantities to be extracted.

Here, 3D fully-resolved DNS of turbulent premixed combustion are perso formed in a spatially-developing slot-burner Bunsen flame configuration with a detailed methane-air chemical mechanism. The first simulation from this study was published in Ref. [11] along with a study of the flame structure and thickness. This study was later expanded to a series of three simulations in the TRZ regime at successively higher turbulence intensities as part of a parametric study. In this paper, we publish those three simulations along with data analysis. The slot jet flame configuration chosen is one of the many configurations that can be used to simulate a stationary premixed flame. Dunstan et al. [12] have compared the influence of the configuration geometry on flame speed statistics using three canonical configurations, namely freely propagating planar flames, stagnation flow planar flames and rod-stabilized V-flames. Their results show that the burning velocity will have a strong dependence on the flame geometry, which itself would depend on the orientation of the shear and flow divergence with respect to the flame surface. The data presented in this paper using a slot jet configuration provides statistics in the presence of strong mean shear that is aligned in roughly the same direction as the mean flame brush. There is also a strong spatial dependence of the flame characteristics due to the downstream development of the jet. Due to these characteristics, the jet configuration chosen in this DNS study and also by Bell et al. [13] are the closest to laboratory premixed 
100

jet flames.

The paper is organized as follows. Sections 2 and 3 presents the problem configuration and numerical method used in the study. This is followed by results and discussions in Section 4 where the data is analyzed to obtain statistical measures of the influence of turbulence on flame structure. In particular the effect of turbulent strain on preheat layer thickness and the integrity of the reaction layers are studied. Also statistical measures of the influence of turbulence on the burning velocity of the flame are presented. The contribution to the burning rate due to wrinkling of the flame $\left(A^{\prime}\right)$ and the enhancement of burning rate per unit surface area $\left(I_{0}\right)$ are determined. 110 Then, the response of the flame surface displacement speed to strain rate and curvature are studied.

\section{Problem Configuration}

The simulation was performed in a slot-burner Bunsen flame configuration. The slot-burner Bunsen configuration is especially interesting due to the presence of mean shear in the flow and is similar in configuration to the burner used in experimental studies, for example by Filatyev et al. [14] and numerical simulated using DNS by Bell et al. [13]. Aside from the similarity in configuration, the parameter space explored in this work is quite different since that flame is within the flamelet regime while the present work is in the TRZ regime. This configuration consists of a central reactant jet through which premixed reactants are supplied. The central jet is surrounded on either side by a heated coflow, whose composition and temperature are those of the complete combustion products of the reactant jet. This arrangement 
is similar to the pilot flame surrounding slot burners commonly used in ex-

1. Flame speed, $S_{\mathrm{L}}=1.8 \mathrm{~m} / \mathrm{s}$

2. Thermal thickness based on maximum temperature gradient, $\delta_{\mathrm{L}}=0.3 \mathrm{~mm}$

3. Full width at half maximum (FWHM) of heat release rate, $\delta_{\mathrm{H}}=0.14 \mathrm{~mm}$, and

4. Flame time scale, $\tau_{\mathrm{f}}=\delta_{\mathrm{L}} / S_{\mathrm{L}}=0.17 \mathrm{~ms}$.

One of the reasons for choosing a preheated inflow condition is that the cost of computation is inversely proportional to the Mach number at the inflow. Preheating the reactants leads to a higher flame speed and allows a higher inflow velocity without blowing out the flame. Also, many practical devices such as internal combustion engines, gas turbines and recirculating furnaces operate at highly preheated conditions. However, such devices also operate under high pressures. We have chosen to perform the simulations at the preheated temperatures for numerical advantages and computational cost savings. High pressure flames are also thinner compared to atmospheric flames. This significantly increases the computational cost of the simulations and hence we could only afford to simulate atmospheric pressure flames. One important consequence of preheating is that the reaction zone is broadened at $800 \mathrm{~K}\left(\delta_{\mathrm{L}} / \delta_{\mathrm{H}}=2\right)$ compared to $300 \mathrm{~K}\left(\delta_{\mathrm{L}} / \delta_{\mathrm{H}}=3\right)$, which is reproduced in the conditions chosen here.

A series of three simulations were performed as a parametric study to investigate the effect of increasing turbulence intensity on lean premixed 
combustion. The problem configuration, mixture equivalence ratio and temperature are the same for all three simulations. However, they differ in the domain sizes and inflow turbulence conditions. The simulation parameters are given in Table 1. A uniform grid spacing of $20 \mu \mathrm{m}$ was used in the streamwise, $x$, and spanwise, $z$, directions, while an algebraically stretched mesh in the transverse, $y$, direction was obtained from $y(s)=f(s) \times L_{\mathrm{y}} / 2$, where $s$ is the equi-spaced computational grid and $0 \leq s \leq 1$. The stretching function is given by,

$$
f(s)=\beta s+\frac{1}{2}\left(1+\tanh \frac{s-s^{*}}{\sigma}\right)\left(e^{k s}-\beta s\right),
$$

where $k=\ln \left(\beta s^{*}\right) /\left(s^{*}-1\right)$. The resultant mesh was mirrored across the jet centerline $(y=s=0)$ to obtain a symmetric mesh. The form of the stretching function along with the choice of constants, $\beta=0.55, s^{*}=0.75$ and $\sigma=1 / 16$, yields a mesh that has a uniform spacing of $20 \mu \mathrm{m}$ in the center of the domain over a region that is five times as wide as the fuel slot at the inflow. The increase in grid spacing, $\left(\Delta_{i+1} / \Delta_{i}-1\right)$, in the outer part of the domain does not exceed $2 \%$. While the uniform grid spacing at the center of the jet ensures numerical fidelity and flexibility in post-processing, the boundaries are pushed farther away to reduce their influence on the flame. The $20 \mu \mathrm{m}$ grid spacing was sufficient to fully resolve all the turbulence scales since the grid spacing was less than twice the kolmogorov scale in all three cases. Also, the flame thickness was equal to fifteen grid spacings thereby allowing sufficient resolution of the flame as well. All the three simulations were also performed at half resolution with a grid spacing of $40 \mu \mathrm{m}$ and the same results were observed, thereby verifying grid convergence. 


\section{Numerical Method}

A reduced chemical mechanism for lean premixed methane-air flames was derived, specifically tailored to minimize temporal stiffness while maintaining

175 
to compute the effective Lewis numbers that were used in the current simulations. Earlier study of lean premixed methane-air flames by Hawkes and Chen [20] found a good agreement between the solutions computed with constant Lewis number transport and mixture-averaged transport over a wide range of strain rates. Furthermore, the Lewis number transport approximation provided considerable computational cost savings that allowed us to invest those resources in pursuing larger mesh sizes and turbulent Reynolds numbers.

A top hat profile function of the form,

$$
f(y)=\frac{1}{2}\left[\tanh \left(\frac{y+h / 2-3 s}{s}\right)-\tanh \left(\frac{y-h / 2+3 s}{s}\right)\right]
$$

is used to specify the mean inflow profiles. The function, $f(y)$ has a value of unity at the center of the jet and zero in the coflow. The transition is smooth and controlled with $s$, which was set to $2.5 \%$ of the jet width. The flame is anchored at the inflow plane by specifying the species mass fractions and temperature from an unstrained laminar flame solution using a progress variable lookup. The progress variable is specified on the inflow plane such that an unburnt mixture issues through the center jet $(c=0)$ and burnt mixture issues through the coflow $(c=1)$. The mixture composition and temperature at the inflow are held constant during the simulation. The mean inflow velocity profile was similarly obtained using the function $f(y)$. A turbulent velocity field was synthesized by specifying the length scale, magnitude of velocity fluctuations and spectral energy density. This velocity field was used as the initial condition for a secondary non-reacting simulation in a temporally evolving streamwise periodic flow configuration. The secondary simulation was advanced in time for one eddy-turn over time to 
achieve sufficient development of the flow field. The resultant flow field was then frozen in time, and injected at the inflow plane in addition to the mean inflow velocity profile.

Navier-Stokes characteristic boundary conditions (NSCBC) [21, 22, 23, 24 were used to prescribe the boundary conditions. The boundary conditions were periodic in the spanwise direction $(z)$, non-reflecting inflow and outflow in the streamwise direction $(x)$, and non-reflecting outflow [23, 24] in the transverse direction $(y)$. Based on the jet inlet velocity and the streamwise domain length, the flow-through time is $0.24 \mathrm{~ms}$ for all three cases. The 230 solution was advanced at a constant 2ns time-step for three flow through times for case A and two flow through times for cases B and C. The first flow through time was neglected from analysis to remove any effects due to initial transients. Data from 61 equally spaced time instants from the remainder of the simulation was used to obtain the statistical results presented in the next section. The fully resolved calculations presented here were preceded by half resolved simulations with a grid spacing of $40 \mu \mathrm{m}$. Since the half resolved simulations cost substantially less, we could afford to advance those calculations much longer in time. An analysis of the half resolved calculations showed that all of the initial transients had disappeared by the end of the first flow through time. The half resolved simulations were also used to verify that data from one flow through time provides sufficient statistics for the analysis that was performed in this paper.

Averaging is performed in the homogeneous direction $(z)$ and time. Symmetry across the centerline is exploited in the statistical analysis where feasi245 ble. Since the flow develops in the streamwise direction, results in this paper 
are presented at selected streamwise locations denoted by the fraction of the domain length, such as $1 / 4,1 / 2$ and $3 / 4$. As shown in Table 1 , the domain lengths and inflow velocities are such that all three cases have a flow through time of $0.24 \mathrm{~ms}$ across the entire domain. The premixed flames are known to have a finite response time to imposed oscillations [25]. The selected streamwise locations correspond to positions with the same flow time from inflow for all the three cases. Specifically, the $1 / 4,1 / 2$ and $3 / 4$ positions correspond to a flow time of $60 \mu \mathrm{s}, 120 \mu \mathrm{s}$ and $180 \mu \mathrm{s}$, respectively. In comparison the flame time scale was $167 \mu \mathrm{s}$. The ratio of flow time to the flame time scale at these three locations equals $0.36,0.72$ and 1.08 , respectively.

\section{Results and Discussion}

\subsection{Turbulence characteristics}

Due to the presence of mean shear in the configuration, the turbulence scales continue to evolve downstream. The development of favre-averaged turbulence intensity along the jet centerline is shown in Fig. 1. It is seen that $u^{\prime}$ quickly decays from the imposed inflow conditions close to the inlet and varies less drastically at further downstream locations. Similarly the turbulence length scales were also observed to re-adjust quickly from those of the synthesized field injected at the inlet. Therefore, the simulations are characterized by the turbulence levels at the $1 / 4$ th downstream location $(x=$ $\left.1 / 4 L_{\mathrm{x}}\right)$ instead of those at the inlet. Table 1 lists the various turbulence characteristics of the flow-field obtained from averaging the flow-field over multiple times. Based on the turbulence parameters the three flames are in the thin reaction zones (TRZ) regime [1] of combustion, progressively moving 
270

from the boundary with the flamelets regime (case A) towards the boundary with the broken reaction zones (BRZ) regime (case $\mathrm{C}$ ). However, case $\mathrm{C}$ is still within the TRZ regime and does not show any indication of broken reaction zones as will be presented later. In comparing cases $\mathrm{B}$ and $\mathrm{C}$, it is seen that the Damkohler number was very similar between these two cases. Since the turbulence intensity and length scale both increased when going from case B to C, the Damkohler number remained fairly constant. It is possible that had the turbulence intensity increased without changes to the length scales, the flame might have gotten closer to the BRZ regime. Since, we are using a spatially evolving jet configuration, the turbulence scales specified at the inflow have only a marginal effect on the actual development of the jet. Therefore, attempting to achieve precise control of the turbulence conditions would be cumbersome.

As seen in Table. 1, the Karlovitz numbers are very high in the current simulations. Also, the ratio of the thermal thickness to the reaction zone thickness is only 2. Therefore, based on Peters' theory [2] one would expect that the transition from the TRZ to BRZ should occur at a Karlovitz number equal to 4 . In spite of having much higher Karlovitz numbers, we have not found any evidence of BRZ in our simulations. There are two possible reasons for this behavior. Firsty, the turbulence parameters were measured in the centerline of the jet at the $1 / 4$ th downstream location. The mixture at the centerline is unreacted and hence at the unburnt temperature. The small scales of turbulence seen here would dissipate quickly before they reach the reaction zone due to the effects of heat release, thermal expansion and increased viscous dissipation. Other studies in the BRZ regime [26] have used 

conditions, the heat release is a maximum at $c=0.65$. 


\subsection{Treatment of flame as an iso-surface}

The progress variable iso-surface, $c=0.65$, is taken as representative of the instantaneous images and also in the instantaneous images at other times 
(not shown). In Fig. 2, Cases $\mathrm{B}$ and $\mathrm{C}$ appear to have holes in the isosurface. These holes are caused due to flame annihilation via self-propagation and are

\subsection{Mean Flame Brush}

Since the instantanous images showed pinch off, it is necessary to verify that the average flame brush was contained within the domain height and also to estimate the amount of reactant leakage. The mean progress variable computed by favre-averaging is shown in Fig. 3 and gives a general picture of the shape and height of the mean flame brush. Firstly it is seen that the flame brush is taller in cases B and C than A. This is due to the higher jet velocity in cases $\mathrm{B}$ and $\mathrm{C}$ compared to $\mathrm{A}$. Secondly it is seen that cases $\mathrm{B}$ and $\mathrm{C}$ have similar flame height inspite of the larger jet width in case B. This gives us the first indication that the net burning velocity in case $\mathrm{C}$ is much higher than case B since a larger flow rate of reactants are being consumed within roughly the same height. It is also seen from these three cases that there is some reactant leakage in the outflow boundary. The leakage is highest in case $\mathrm{A}$, followed by case $\mathrm{C}$ and is the lowest in case B. The amount of reactant leakage due to flame pinch off will be quantified later in this section while computing the burning velocity. Figure 3 also shows the iso-contour of $\tilde{c}=0.65$ that denotes the flame surface location in the mean flame brush and that it was entirely contained within the domain.

\subsection{Effect of turbulence on flame thickness}

365 As mentioned in the introduction, the effect of turbulent stirring on the flame thickness in the TRZ regime is not entirely understood. Here, the 
data from the DNS is analyzed to determine if, on average, the flame thickness increases or decreases relative to a laminar flame. The reciprocal of the magnitude of progress variable gradient, $1 /|\nabla c|$ yields a flame thickness analogous to the definition used for the laminar thermal thickness $\delta_{\mathrm{L}} \cdot|\nabla c|$ is averaged over intervals of $\mathrm{c}$ and compared with the unstrained laminar flame profile in Fig. 4. In ref. [11, the conditional mean of $|\nabla c|$ was presented for case $\mathrm{A}$ and the results showed that the mean $|\nabla c|$ was lower in the turbulent flame than in a laminar flame, which indicated flame thickening. Here, the same analysis is also applied to cases $\mathrm{B}$ and $\mathrm{C}$, to verify if the flame continues to get thicker as the turbulence intensity is increased. A comparison of case A with case B in Fig. 4 shows that the mean gradients are further reduced. This again indicates an increase in flame thickening due to the increase in turbulence intensity from $u^{\prime} / S_{\mathrm{L}}=3$ to 6 . However, a comparison of case B with case $\mathrm{C}$ shows that there is negligible increase in flame thickness even though the turbulence intensity was increased from $u^{\prime} / S_{\mathrm{L}}=6$ to 10 . This is a very interesting result and shows that a further increase in turbulence intensity beyond a threshold level might not result in any more thicker flames in the TRZ regime.

In ref. [11], the effect on flame thickness was analyzed using the gradient of progress variable alone. In this paper, the thickening of the flame is further validated using additional analysis based on a distance function. A distance function $D$ is defined such that at every point in the domain the quantity $D$ is the shortest normal distance from the flame-surface [29, 30]. The distance 390 function is computed by solving the Eikonal equation,

$$
|\nabla D|=1
$$


subject to the boundary condition $D=0$ on the flame surface. The flame surface is defined as the isosurface $c=0.65$ as mentioned earlier and the distance to this surface, $D$, is computed at every point in the domain. The distance is taken as negative in the fresh gas side and positive in the burned gas side. An illustration of the distance function versus the progress variable iso-surface is shown in Fig. 5 .

The figure shows a slice perpendicular to the streamwise direction. The progress variable is shown in color and the iso-contours of the computed distance function are shown as line contours. The distance function isocontours represent the locus of all points that are equidistant from the flame surface, $D=0$. Where the flame surface is curved, the distance function is discontinuous on the center of curvature side and has a ridge-like appearance. The ridge is the locus of all points that are equidistant from multiple points on the flame surface [29]. In the absence of any influence from turbulence on the flame structure, as one would expect in the flamelet regime, the isocontours of progress variable $(c)$ and distance function $(D)$ would have been mostly parallel to each other. Figure 5 shows that the flame structure in the preheat layer is considerably disrupted due to small scale turbulent stirring thereby confirming that the flame structure is not flamelet-like at the conditions chosen for these simulations. Due to the effect of stirring on the progress variable ahead of the flame surface, the distance from the flame surface is an additional and possibly better choice of conditioning variable for investigating the flame structure as opposed to conditioning the statistics on progress variable. Therefore, the effect of turbulence on the flame structure is analyzed ${ }_{415}$ by obtaining statistical means conditioned on the distance from the flame 
surface.

Figure 6 shows the mean and standard deviation of temperature and $\mathrm{O}_{2}$ mass fraction as a function of the distance function for cases $\mathrm{A}, \mathrm{B}$ and $\mathrm{C}$ at the ' $1 / 2$ ' streamwise location. Since the progress variable, $c$, is defined based on $\mathrm{O}_{2}$ mass fraction, the $Y_{\mathrm{O}_{2}}$ results are also indicative of the mean and standard deviation of $c$. It is seen from the mean temperature profile that the preheat layer of the flame is significantly thickened. The mean temperature ahead of the flame is higher indicating that there is an enhanced activity in the preheat layer leading to an increased amount of heat diffusion into the unburnt gas region. This is also evident from the mean $\mathrm{O}_{2}$ mass fraction profiles, where $\mathrm{O}_{2}$ is depleted in the preheat layer due to its transport into the reaction zone. However comparing cases B and C, no significant difference is seen in the mean profiles. This is in agreement with the finding based on Fig. 4 that the higher turbulence intensity in case $\mathrm{C}$ compared to case B did not further increase the mean flame thickness.

Figure 6 also shows the standard deviation of temperature and $\mathrm{O}_{2}$ mass fraction for the three cases. It should be noted that since the distance is computed from the $Y_{\mathrm{O} 2}$ iso-surface, the standard deviation of $Y_{\mathrm{O} 2}$ is zero at $D=0$ by definition. $\mathrm{O}_{2}$ mass fraction is strongly correlated with temperature, hence its choice as the progress variable. Therefore the standard deviation of temperature is also very low near $D=0$. Therefore, the dips seen in the standard deviations of temperature and $Y_{\mathrm{O} 2}$ near $D=0$ are not an anomaly. Instead, it is a consequence of the definition used for identifying the flame surface and is to be expected. However, ahead in the fresh gas re440 gion a comparison of the standard deviations among the three cases provides 
very useful information. It is seen that the standard deviation increases with turbulence intensity. It is also seen that comparing cases $\mathrm{B}$ and $\mathrm{C}$ there is a small increase in the level of fluctuations. This suggests that while the mean profiles are similar for cases B and C, differences appear in higher moments.

445

\subsection{Effect on reaction layer}

In Ref. [1], the mean reaction rates conditioned on the progress variable were compared to the laminar reaction rate profiles to study the influence of turbulence on the reaction zone structure. Here we extend the analysis to cases B and C. Also, instead of using $c$ as the conditioning variable, we use the distance variable $D$ to obtain conditional mean reaction rate profiles. Figure 7 shows the mean heat release rate profile and mean reaction rate profiles for $\mathrm{CH}_{4}$ and $\mathrm{OH}$ at the $1 / 2$ streamwise location. Also shown are laminar strained flame profiles computed using OPPDIF [31] using a backto-back counterflow premixed flame configuration. The laminar flame profiles correspond to a strain rate equal to $1.2 / \tau_{\mathrm{f}}$. This strain rate value was chosen because it provided the profiles closest to the DNS results in Fig. 7 .

From Fig. 7, it is seen that the three cases A, B and C show excellent agreement with each other and with the strained laminar flame profile. As will be shown later, the strain rate is significantly higher for cases B and C compared to case A. However, all the three cases have very similar heat release and reaction rate profiles. In contrast to the disruption observed in the preheat layer in figures 4 and 6, we do not find any impact on the reaction layer. The agreement is even better than what was observed in our previous work [11] where the reaction rates were conditioned on progress variable. These results confirm the hypothesis [1] that turbulence does not 
have a significant influence on the reaction zone even in the TRZ regime. Although the reaction zone here is not really 'thin' $\left(\delta_{\mathrm{L}} / \delta_{\mathrm{H}}=2\right)$, the lack of significant influence on the reaction layer could not have been due to length scale differences as originally suggested in Ref. [1] but could be due to other factors such as the dissipation of turbulence due to heat release.

\subsection{Strain rate}

The tangential strain rate, $a_{\mathrm{t}}$, on a flame surface has a significant effect on the burning rate of the flame and surface area generation. The tangential strain rate is computed on two progress variable iso-surfaces - one corresponding to the fresh mixture $(c=0.2)$ and the other corresponding to the approximate location of the heat release layer $(c=0.65)$. Figure 8 shows the evolution of conditional mean strain rate on these two iso-surfaces as a function of the axial distance. The strain rate is higher in cases $\mathrm{B}$ and $\mathrm{C}$ than case A due to the larger jet velocity and the higher mean shear. It is also seen that the mean strain rate decays with distance due to a widening of the shear layer. An important observation is that, in the first, roughly, $1 / 4$ th of the domain, the strain rate at $c=0.2$ is higher than at 0.65 . But this difference disappears at the downstream locations and the mean strain rate is the same at $c=0.2$ and 0.65 . This is because, the flame is initially anchored with the shear layer at the inflow. But, as we proceed downstream, the jet widens, while the flame moves inside the shear layer and the flame tip closes. Therefore the large difference in mean strain rate between $c=0.2$ and $c=0.65$ isosurfaces, disappears. 


\subsection{Global burning velocity}

490

Here, we define and measure a global burning velocity of the flame similar to Filatyev et al. [14. As a first step, we treat the mean turbulent flame as the surface defined by $\tilde{c}=0.65$ as shown in Fig. 3. In our simulations, the domain was large enough that a significant fraction of the reactants that entered the domain were consumed by combustion before exiting the computational domain. A mass flux analysis to measure the mass of fuel entering through the inlet and leaving unburnt from the outflow boundaries showed that $15 \%, 5 \%$ and $9 \%$ of the reactants leave the boundary unburnt in cases A, B and C, respectively. Flame pinch-off, which causes a portion of the flame surface to detach and to be swept downstream due to advection is the main source of unburnt fuel leaving the computational domain. The domain length required to compute the complete combustion of the fuel will make the simulation prohibitively expensive due to flame pinch-off. Therefore, the domain lengths were chosen using estimates of the flame height obtained from lower resolution calculations, and the domain was sized to be long enough to have $90 \%$ of the combustion occur within the domain. Case A was the first case simulated in the series, and our estimate of the flame height was slightly off resulting in $15 \%$ fuel leaving the domain unburnt. In the latter cases, the domain length was long enough to cover over $90 \%$ of combustion. Since the mean flame brush defined by $\tilde{c}=0.65$ is well within the domain, the global burning velocity can be properly estimated inspite of the small mass efflux.

The mass inflow of the reactants is computed by integrating over the inflow plane as,

$$
\dot{m}_{i n}=\int_{A} \rho u_{1} c d A
$$


A turbulent burning velocity $S_{\mathrm{T}}$ can be defined such that, the reactants are consumed at this rate, uniformly, over the area of the mean flame brush

515 measured from Fig. 3. Then the area of this unwrinkled flame is $A_{\text {flat }}=P L_{\mathrm{z}}$, where $L_{\mathrm{z}}$ is the domain length in the spanwise direction. Through mass balance,

$$
\rho_{\mathrm{u}} S_{\mathrm{T}} A_{\text {flat }}=\dot{m}_{\text {in }}
$$

Table 5 shows the $S_{\mathrm{T}}$ computed using the relation 7 along with the ob520 served flame heights for the three cases. It is seen that the ratio of the turbulent burning velocity $\left(S_{\mathrm{T}}\right)$ to the laminar burning velocity progressively increases from case A to C. This is evident from comparing the flame heights alone for cases B and C. Although the jet in case $\mathrm{C}$ was 1.5 times wider than case $\mathrm{B}$, the flame heights were the same. The burning velocity had to be much larger in order to combust the higher mass flux within the same flame height.

Equation 7 was useful to obtain a global burning velocity estimate for the three flames. It is possible to further extend this simple analysis to determine the contributions from increase in flame surface area and from the increase 530 Rewriting Eq. 7]as,

$$
\rho_{\mathrm{u}} S_{\mathrm{L}} I_{0} A_{\text {wrinkled }}=\dot{m}_{i n}
$$

Let $A^{\prime}=A_{\text {wrinkled }} / A_{\text {flat }}$. Then,

$$
\frac{S_{\mathrm{T}}}{S_{\mathrm{L}}}=I_{0} A^{\prime}
$$

The area of the wrinkled flame surface, $A_{\text {wrinkled }}$, is obtained by trian- 
gulation of the instantaneous fields, as in Fig. 2 followed by averaging over

multiple time snapshots. The resultant time averaged wrinkled surface area, $A_{\text {wrinkled }}$, is then normalized using the area of the time-averaged flame brush, $A_{\text {flat }}$, computed in Eq. 7 to obtain $A^{\prime}$. Given $S_{\mathrm{T}} / S_{\mathrm{L}}$ of the mean flame brush and $A^{\prime}, I_{0}$ is computed using Eq. 9. The results are reported in Table 5. It is seen from the area ratios that the extent of wrinkling increases progressively going from case A to case C. However, $I_{0}$ decreases from case A to case B, but is relatively constant between cases B and C.

\subsection{Local burning velocity}

In this section, we perform a more local analysis of the burning velocity and use it to compute $I_{0}$ and $A^{\prime}$ at various downstream locations. First, Eq. 8 is rewritten as,

$$
\rho_{\mathrm{u}} S_{\mathrm{L}} I_{0} A_{\text {wrinkled }}=\int_{0}^{L_{x}} \int_{0}^{L_{y}} \int_{0}^{L_{z}} \dot{\omega}_{\mathrm{c}} d z d y d x
$$

where the mass influx of reactants has been replaced by the volume integrated consumption rate of reactant. For the purpose of this analysis, the domain is decomposed into thin slices of thickness $\Delta$ perpendicular to the streamwise $(x)$ direction. A local balance between the consumption of reactants and turbulent flame propagation is assumed in each slice and a local burning velocity is computed in each $y-z$ slice. Then, Eq. 10 is rewritten as a balance equation for each $y-z$ slice as,

$$
\rho_{\mathrm{u}} S_{\mathrm{L}} I_{0}(x) A_{\text {wrinkled }}(x)=\int_{x}^{x+\Delta} \int_{0}^{L_{y}} \int_{0}^{L_{z}} \dot{\omega}_{\mathrm{c}} d z d y d x^{\prime}
$$

where $A_{\text {wrinkled }}(x)$ is the flame surface area within a local slab of thickness $\Delta$ at location $x$. On substituting $A_{\text {wrinkled }}(x)$ in Eq. 11 , the local burning ef- 
555

\subsection{Displacement speed}

In numerical studies with finite rate chemistry, a mathematically rigorous definition of the flame burning velocity, known as the displacement speed $\left(S_{\mathrm{d}}\right)$, is well suited. The displacement speed is defined as the velocity of the 
flame iso-surface with respect to the local fluid medium [32] and is given by

$$
S_{\mathrm{d}}=-\frac{D c}{D t} \frac{1}{|\nabla c|}
$$

where the negative sign ensures that the displacement speed is positive when a reactant that is consumed in combustion is used to evaluate $S_{\mathrm{d}}$, as is the case here. $S_{\mathrm{d}}$ can be decomposed in to three components [33] as,

$$
S_{\mathrm{d}}=S_{\mathrm{d}, \mathrm{r}}+S_{\mathrm{d}, \mathrm{n}}+S_{\mathrm{d}, \mathrm{c}}
$$

where the three terms on the right hand side are the reaction, normal diffusion and tangential diffusion terms respectively. Further, a density weighted displacement speed is defined as $S_{\mathrm{d}}^{*}=\rho S_{\mathrm{d}} / \rho_{\mathrm{u}}$ where $\rho_{\mathrm{u}}$ is the unburnt mixture density. Weighting by the local density allows the displacement speed to be measured with respect to the unburnt mixture conditions as is customary for other definitions of flame speed.

Here, a conditional average of $S_{\mathrm{d}}^{*}$ is computed on the $c=0.65$ iso-surface and plotted as a function of axial distance in Fig. 10. It is seen that the mean displacement speed increases with downstream direction for all 3 cases, similar to the trend shown by $I_{0}$ in Fig. 9. It is also seen that mean $S_{\mathrm{d}}^{*}$ is negative close to the inlet for cases B and C. Premixed flames are known to have a negative displacement speed at large strain rates. This has been observed in opposed flow experiments with a reactant and burnt product stream opposed to each other. Under high strain rates, It has been observed that the premixed flame can move from the reactant side past the stagnation plane in to the product stream and exhibit a negative displacement speed.

Here too, the negative displacement speed can be attributed to the very high strain rates observed close to the inlet. 
It is important to contrast the displacement speed of the flame surface from the burning velocities computed in sections 4.8 and 4.9 which were based on a balance equation for the reactant consumption rate. The displacement

\subsection{Dependence of flame speed on strain rate}

In this section, we analyze the strain rate and displacement speed statistics on the $c=0.65$ isosurface at the various downstream locations. Figure 11 shows the distribution of strain rates on the flame surface. The probability distribution function (pdf) of strain rate shows that the width of the strain rate distribution decreases in the downstream direction for all 3 cases presumably due to the jet development. It is also seen that cases $\mathrm{B}$ and $\mathrm{C}$ have a much larger distribution of strain rates compared to case A. The more turbulent cases have a significantly higher probability for larger strain rates on the flame surface. While the distributions for cases B and C look similar at the $1 / 4 L_{x}$ location, they start to show differences in the downstream locations.

Next, we look at the strain rate conditional mean displacement speeds computed on the flame surface at different downstream locations. These results are shown in Fig. 12. Also the response of a laminar strained flame 
in a opposed flow configuration with a reactant streams opposed to a burnt product stream was computed using OPPDIF [31] and is shown in Fig.. 12 for reference. The slope of the conditional means of the displacement speed to the strain rate is also known as the Markstein length. It is seen that the slope is lower in turbulent flames in the TRZ regime compared to the laminar strained flame. It is also seen that the displacement speed has a positive slope for negative strain rates, implying that, on an average, compressive strains tend to reduce the displacement speed.

\subsection{Dependence of flame speed on curvature}

Here, the correlation of displacement speed and its components to curvature is analyzed. Figure 13 shows the distribution of curvatures on the $c=0.65$ iso-surface at the $x=1 / 2 L_{x}$ location. It is seen that the curvature distribution for all 3 cases looks similar with only minor differences. It is seen that the curvature distribution is not symmetric and the distribution has a longer tail towards the positive curvature side. This shows that flames with very high positive curvatures (center of curvature is in the burnt gas) are more likely than those with very high negative curvatures. This quantitatively confirms the observation made based on Fig. 2 that the surface forms small radius cusps when the center of curvature is in the burnt gas. We need to confirm that this shape of the flame surface originates from flow induced straining as opposed to Huygens-type self propagation of a surface. To confirm that, we compute the mean curvature conditioned on strain rate on the $c=0.65$ isosurface and present the distribution at the $x=1 / 2 L_{x}$ location in Fig. 14. It is seen that the mean curvature at positive strain rates is slightly negative. However, at negative strain rates, the mean curvature 
quickly increases to large positive values. This indicates that compressive strain conditions cause the flames to form sharp cusps with the center of curvature on the burnt gas side, thereby confirming that the cusp formation is due to flow strain effects.

Next we study the effect of curvature on the propagation velocity of the flame. The average of $S_{\mathrm{d}}^{*}$ conditioned on curvature and $c=0.65$ is shown in Fig. 15 for all three cases. Also, the correlation of the components of $S_{\mathrm{d}}^{*}$ namely reaction, normal diffusion and curvature or tangential diffusion is shown in Fig. 16 for case C (cases A and B had a similar correlation as case $\mathrm{C}$ and hence omitted). All three cases show a similar response to curvature with very little variation between the 3 cases. It is also seen that $S_{\mathrm{d}}^{*}$ shows a non-linear response to curvature with two distinct slopes - a higher slope on the negative curvature side and a lower slope on the positive curvature side. There are some similarities with previously observed results by Echekki et al. [33] which was based on a two-dimensional DNS in the flamelet regime. The reaction term has the largest contribution to the net displacement speed and also shows a two-slope correlation to curvature with a negative slope at negative curvatures and zero slope at positive curvatures. 670 The non-linearity in the correlation of net $S_{\mathrm{d}}^{*}$ can be attributed to the reaction term. The tangential diffusion term correlates negatively and linearly with curvature as is to be expected. Normal diffusion term is very small and has a negligible contribution to the net displacement speed. Finally, it is confirmed that negative displacement speeds (flame receding) are possible at 675 large positive curvatures mainly due to the effect of the tangential diffusion term. 


\section{Conclusions}

Three dimensional direct numerical simulation of a spatially-developing slot Bunsen flame at successively higher turbulence intensities was presented. These simulations were performed at conditions that are in the TRZ regime of premixed combustion. That the conditions are in the TRZ regime was confirmed through an analysis of the flame structure that showed a thickening of the flame, turbulent stirring and disruption of the preheat layer structure, but minimal impact on the reaction zone. Global and local statistics for the burning velocity and flame surface area were computed and its dependence on strain rate and curvature were studied. The results show an increased flame surface area generation with increasing turbulence intensity. However, the increase in burning rate per unit area was seen to asymptote for the last two cases. The dependence of the flame displacement speed on strain rate showed that the Markstein length was similar for the three cases and were all lower than those seen in the laminar strained flames. Cases B and $\mathrm{C}$ tend to be similar, while case $\mathrm{A}$ had a lower mean displacement speed conditioned on strain rate than the other two cases. On the contrary, all the three cases had similar responses to curvature. The asymptoting of some of the key quantities between cases B and C indicates that the flame structure, especially the reaction zone, is more immune to the effects of turbulence than previously thought. The transition from TRZ to BRZ regime would require considerably higher turbulence intensities than predicted in the regime diagrams based on theoretical studies. The transition point between these two regimes needs to be determined by further studies at higher turbulence intensities and Karlovitz numbers. It is envisioned that such simulations will 
also provide valuable data for model development and validation in both the TRZ and BRZ regimes.

\section{Acknowledgments}

705 cility at the Oak Ridge National Laboratory, which is supported by the Office of Science of the U.S. Department of Energy under Contract No. DE-AC0500OR22725. The work at SNL was supported by the Division of Chemical Sciences, Geosciences and Biosciences, the Office of Basic Energy Sciences 710 BES, SciDAC Computational Chemistry program. SNL is a multiprogram laboratory operated by Sandia Corporation, a Lockheed Martin Company, for the U.S. DOE under contract DE-AC04-94-AL85000.

\section{References}

[1] N. Peters, Journal of Fluid Mechanics 384 (1999) 107-132.

[2] N. Peters, Turbulent Combustion, Cambridge university press, New York, 2000.

[3] M. S. Mansour, N. Peters, Y. C. Chen, Proceedings of the Combustion Institute 27 (1998) 767-773.

${ }_{720}^{20}[4]$ Y. C. Chen, M. S. Mansour, Proceedings of the Combustion Institute 27 (1998) 811-818. 
[5] F. Dinkelacker, A. Soika, D. Most, D. Hofmann, A. Leipertz, W. Polifke, K. Dobbeling, Proceedings of the Combustion Institute 27 (1998) 857865 .

725

[12] T. D. Dunstan, N. Swaminathan, K. N. C. Bray, Journal of Fluid Mechanics 709 (2012) 191-222.

[13] J. B. Bell, M. S. Day, J. F. Grcar, M. J. Lijewski, J. F. Driscoll, S. A. Filatyev, Proceedings of the Combustion Institute 31 (2007) 1299-1307.

740 [14] S. A. Filatyev, J. F. Driscoll, C. D. Carter, J. M. Donbar, Combustion and Flame 141 (2005) 1-21. 
[15] R. J. Kee, J. F. Grcar, M. D. Smooke, J. A. Miller, PREMIX: A Fortran Program for Modeling Steady Laminar One-Dimensional Premixed Flames, Technical Report SAND85-8240, Sandia National Laboratories, 1985.

[16] R. Sankaran, E. R. Hawkes, J. H. Chen, T. Lu, C. K. Law, in: Proceedings of the 44nd Aerospace Sciences Meeting and Exhibit, Reno, USA. AIAA Paper 2006-0165.

[17] C. A. Kennedy, M. H. Carpenter, Applied Numerical Mathematics 14 (1994) 367-458.

[18] C. A. Kennedy, M. H. Carpenter, R. M. Lewis, Applied Numerical Mathematics 35 (2000) 177-264.

[19] R. J. Kee, F. M. Rupley, J. A. Miller, Chemkin-II: A Fortran Chemical Kinetics Package for the Analysis of Gas-Phase Chemical Kinetics, Technical Report SAND89-8009B, Sandia National Laboratories, 1991.

[20] E. R. Hawkes, J. H. Chen, Combustion and Flame 144 (2006) 112-125.

[21] T. J. Poinsot, S. K. Lele, Journal of Computational Physics 101 (1992) 104-129.

[22] J. C. Sutherland, C. A. Kennedy, Journal of Computational Physics 191 (2003) 502-524.

[23] C. S. Yoo, Y. Wang, A. Trouve, H. G. Im, Combustion Theory and Modeling 9 (2005) 617-646. 
[24] C. S. Yoo, H. G. Im, Combustion Theory and Modeling 11 (2007) 259286.

765

[25] H. G. Im, J. H. Chen, Proceedings of the Combustion Institute 28 (2000) 1833-1840.

[26] B. Savard, B. Bobbitt, G. Blanquart, Proceedings of the Combustion Institute 35 (2015) 1377-1384.

[27] W. E. Lorensen, H. E. Cline, in: Proceedings of the 14th Annual Con770

[28] S. B. Pope, Int J Eng Science 26 (1988) 445-469.

[29] J. A. Sethian, SIAM Review 41 (1999) 199-235.

[30] T. Deschamps, L. D. Cohen, Medical Image Analysis 5 (2001) 281-299.

775 [31] A. E. Lutz, R. J. Kee, J. F. Grcar, F. M. Rupley, OPPDIF: A Fortran Program for Computing Opposed-Flow Diffusion Flames, Technical Report SAND96-8243, Sandia National Laboratories, 1997.

[32] T. Echekki, J. H. Chen, Combustion and Flame 106 (1996) 184-202.

[33] T. Echekki, J. H. Chen, Combustion and Flame 118 (1999) 308-311. 
780 List of Tables

$1 \quad$ Simulation parameters . . . . . . . . . . . . . . . . 39

口 2 Species-wise Lewis numbers used in the molecular transport

model. . . . . . . . . . . . . . . . . . 40

$3 \quad$ Global burning velocity of the turbulent flame. . . . . . . . . . 41 


\section{List of Figures}

a 1 Favre-averaged turbulence intensity $\left(u^{\prime} / S_{\mathrm{L}}\right)$ along the jet cen-

terline. . . . . . . . . . . . . . . . . . . 42

a 2 Instantaneous iso-contour of the progress variable $(c=0.65)$

representing flame surface for cases A, B and C. . . . . . . . . 43

790 ․ $3 \quad$ Favre averaged mean progress variable $(\tilde{c})$ is shown as a pseu-

docolor plot. The color scale varies from blue (0) to red (1).

The iso-contour of $\tilde{c}=0.65$ is shown as a solid line. . . . . . . . 44

․ 4 Conditional means of $|\nabla c|$ compared to the laminar flame pro-

file for the three simulations. The means are computed at

795 three chosen streamwise locations corresponding to $1 / 4,1 / 2$

and 3/4th of the domain length in the stream-wise direction. 45

\ 5 Instantaneous progress variable $(c)$ and distance function $(D)$

are shown in a slice of the domain perpendicular to the stream-

wise direction for case C. The color contours show $c$ on a blue

$800 \quad(c=0)$ to red $(c=1)$ scale. The lines represent iso-contours of

$D$. The darkest iso-contour line represents $D=0$, which also

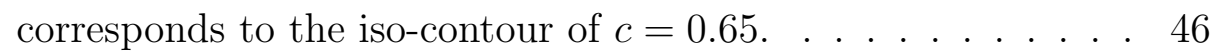

a 6 Mean and standard deviation of temperature and $Y_{\mathrm{O} 2}$ con-

ditioned on the distance from the flame surface. Means are

$805 \quad$ computed at the streamwise location ' $1 / 2$ '. . . . . . . . . 47 
- 7 Mean heat release rate and reaction rates conditioned on the

distance from the flame surface compared to the laminar pro-

file. Results shown are at the $1 / 2$ streamwise location. The

laminar flame results correspond to a strained laminar flame

810 solution at strain rate $a_{\mathrm{t}} \tau_{\mathrm{f}}=1.2 \quad \ldots \ldots \ldots$

8 Mean strain rate, conditioned on $c$, as a function of the axial

distance. In (a) $c=0.2$ and in (b) $c=0.65$. . . . . . . . 49

a $9 \quad$ Flame area $\left(A^{\prime}\right)$ and burning efficiency $\left(I_{0}\right)$ variation along the axial direction . . . . . . . . . . . . . . . . 50

815 a $10 \quad$ Mean $S_{\mathrm{d}}^{*}$, conditioned on $c=0.65$, as a function of the axial distance. . . . . . . . . . . . . . . . . 51

$11 \quad$ PDF of strain rate conditioned on $c=0.65$ at the $1 / 4,1 / 2$ and $3 / 4$ downstream locations. . . . . . . . . . . . . 52

12 Strain-rate conditional PDF of the displacement speeds on the 820 \begin{tabular}{|c|}
\hline$c=0.65$ isosurface at $1 / 4,1 / 2$ and $3 / 4$ downstream locations. \\
53
\end{tabular}

13 PDF of curvature conditioned on $\mathrm{c}=0.65$ at the $1 / 2$ downstream location. . . . . . . . . . . . . . . . . . . . . 54

- 14 Strain-rate conditional mean of curvature on $c=0.65$ surface at the $1 / 2$ downstream location. . . . . . . . . . . 55

825 व 15 Curvature conditional mean $S_{\mathrm{d}}^{*}$ on $\mathrm{c}=0.65$ at the $1 / 2$ downstream location. . . . . . . . . . . . . . . . . . . . 56

16 Curvature conditional mean $S_{\mathrm{d}}^{*}$ components on $\mathrm{c}=0.65$ at the $1 / 2$ downstream location for case C. . . . . . . . . 57 
Table 1: Simulation parameters

\begin{tabular}{c|c|c|c} 
& Case A & Case B & Case C \\
\hline \hline Slot width $(h)$ & $1.2 \mathrm{~mm}$ & $1.2 \mathrm{~mm}$ & $1.8 \mathrm{~mm}$ \\
Domain size, $L_{x} \times L_{y} \times L_{z}$ & $12 h \times 12 h \times 3 h$ & $20 h \times 12 h \times 3 h$ & $13 h \times 12 h \times 3 h$ \\
Mesh size, $N_{x} \times N_{y} \times N_{z}$ & $720 \times 400 \times 180$ & $1200 \times 400 \times 180$ & $1200 \times 600 \times 270$ \\
Number of grid points & 52 Million & 86 Million & $195 \mathrm{Million}$ \\
\hline Turbulent jet velocity $(\bar{U})$ & $60 \mathrm{~m} / \mathrm{s}$ & $100 \mathrm{~m} / \mathrm{s}$ & $100 \mathrm{~m} / \mathrm{s}$ \\
Laminar coflow velocity & $15 \mathrm{~m} / \mathrm{s}$ & $25 \mathrm{~m} / \mathrm{s}$ & $25 \mathrm{~m} / \mathrm{s}$ \\
Turbulence intensity ${ }^{4}\left(u^{\prime} / S_{\mathrm{L}}\right)$ & 3 & 6 & 10 \\
Turbulence length scale ${ }^{2,4}\left(l_{\mathrm{t}} / \delta_{\mathrm{L}}\right)$ & 0.7 & 1 & 1.5 \\
Integral length scale ${ }^{3,4}\left(l_{33} / \delta_{\mathrm{L}}\right)$ & 2 & 2 & 4 \\
\hline Jet Reynolds no $\left(\operatorname{Re}_{\text {jet }}=\bar{U} h / \nu\right)$ & 840 & 1400 & 2100 \\
Turbulence Reynolds no $\left(\mathrm{Re}_{\mathrm{t}}=u^{\prime} l_{33} / \nu\right)$ & 40 & 75 & 250 \\
Karlovitz no $\left(\delta_{\mathrm{L}} / l_{\mathrm{k}}\right)^{2,5}$ & 100 & 225 & 400 \\
Damkohler no $\left(S_{\mathrm{L}} l_{33} / u^{\prime} \delta_{\mathrm{L}}\right)$ & 0.7 & 0.3 & 0.4 \\
\hline \hline
\end{tabular}

${ }^{1}$ Kinematic viscosity at the inflow conditions, $\nu=8.5 \times 10^{-5} \mathrm{~m}^{2} / \mathrm{s}$, is used to compute Reynolds number.

${ }^{2}$ Turbulence length scale, $l_{\mathrm{t}}$, is estimated as $l_{\mathrm{t}}=u^{\prime 3} / \tilde{\epsilon}$, where $\tilde{\epsilon}$ is the average turbulent kinetic energy dissipation rate.

${ }^{3}$ Integral length scale, $l_{33}$, is defined as the integral of the auto- correlation of the spanwise component of velocity in the spanwise direction.

${ }^{4}$ The turbulence scales evolve from the synthetic turbulence specified at the inflow. The $u^{\prime}, l_{\mathrm{t}}$ and $l_{33}$ values reported here are at the $1 / 4$ th streamwise location along the jet centerline.

${ }^{5}$ Kolmogorov length scale, $l_{k}$, is estimated as $\left(\nu^{3} / \tilde{\epsilon}\right)^{1 / 4}$ 
Table 2: Species-wise Lewis numbers used in the molecular transport model.

\begin{tabular}{c|c} 
Species & Lewis No. \\
\hline $\mathrm{H}_{2}$ & 0.29 \\
$\mathrm{H}$ & 0.17 \\
$\mathrm{O}$ & 0.69 \\
$\mathrm{O}_{2}$ & 1.08 \\
$\mathrm{OH}$ & 0.70 \\
$\mathrm{H}_{2} \mathrm{O}$ & 0.82 \\
$\mathrm{HO}_{2}$ & 1.07 \\
$\mathrm{CH}_{3}$ & 0.97 \\
$\mathrm{CH}_{4}$ & 0.96 \\
$\mathrm{CO}_{2}$ & 1.07 \\
$\mathrm{CO}_{2}$ & 1.34 \\
$\mathrm{CH}_{2} \mathrm{O}$ & 1.25 \\
$\mathrm{~N}_{2}$ & 1.04
\end{tabular}


Table 3: Global burning velocity of the turbulent flame.

\begin{tabular}{c|c|c|c} 
& Case A & Case B & Case C \\
\hline Flame height $(H)$ & $13.3 \mathrm{~mm}$ & $18.1 \mathrm{~mm}$ & $18.7 \mathrm{~mm}$ \\
\hline$S_{\mathrm{T}} / S_{\mathrm{L}}$ & 1.47 & 1.9 & 2.67 \\
\hline$A^{\prime}$ & 1.5 & 2.2 & 3.0 \\
\hline$I_{0}$ & 0.98 & 0.86 & 0.89 \\
\hline
\end{tabular}




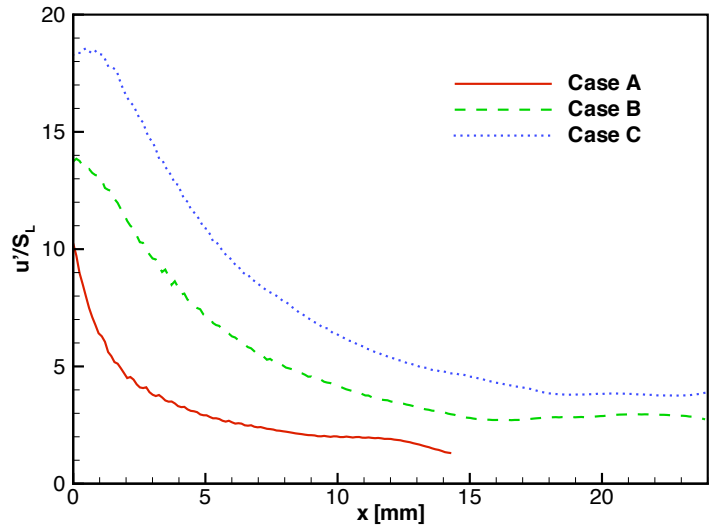

Figure 1: Favre-averaged turbulence intensity $\left(u^{\prime} / S_{\mathrm{L}}\right)$ along the jet centerline. 


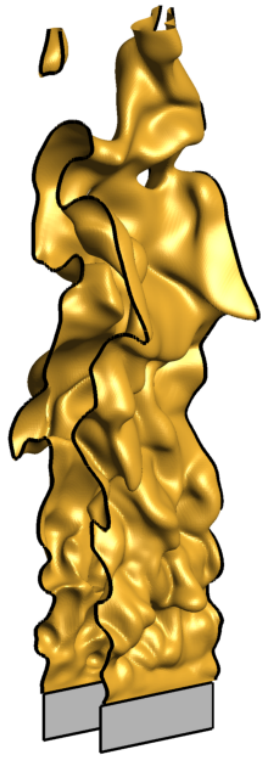

Case A

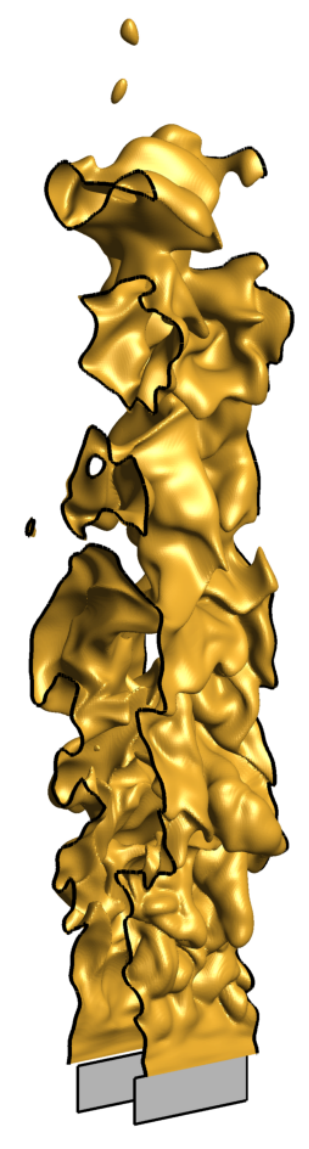

Case B

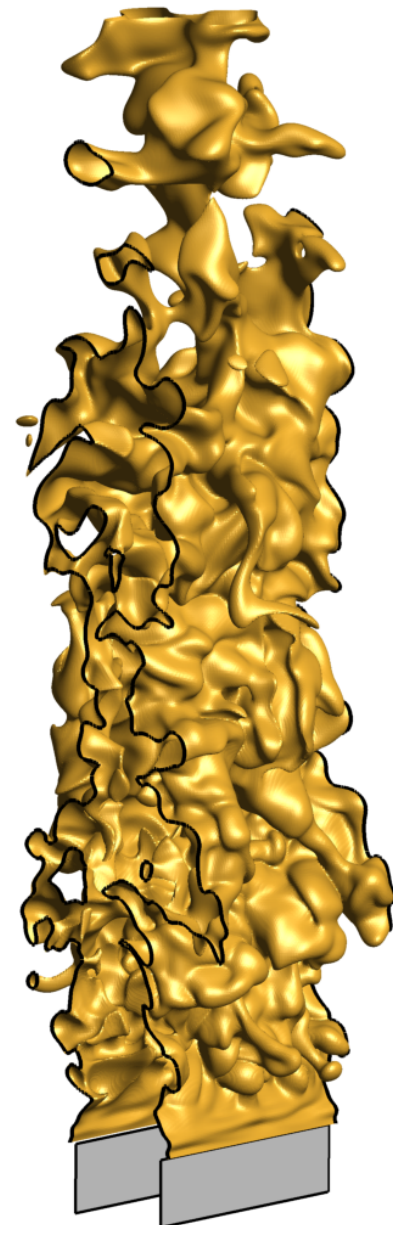

Case C

Figure 2: Instantaneous iso-contour of the progress variable $(c=0.65)$ representing flame surface for cases A, B and C. 


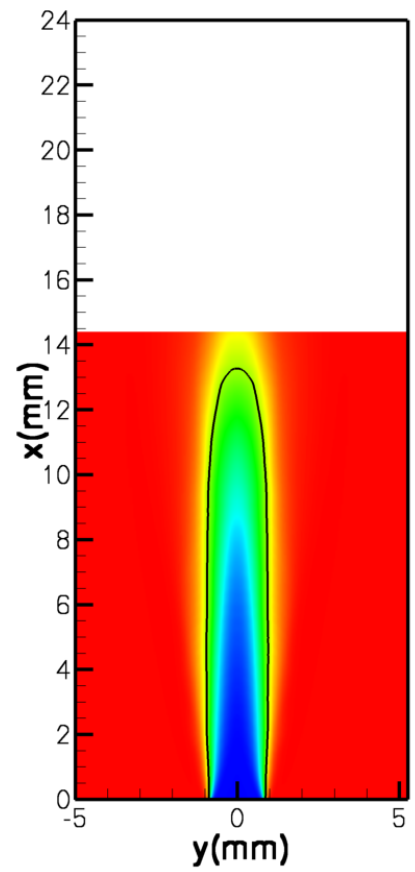

Case A

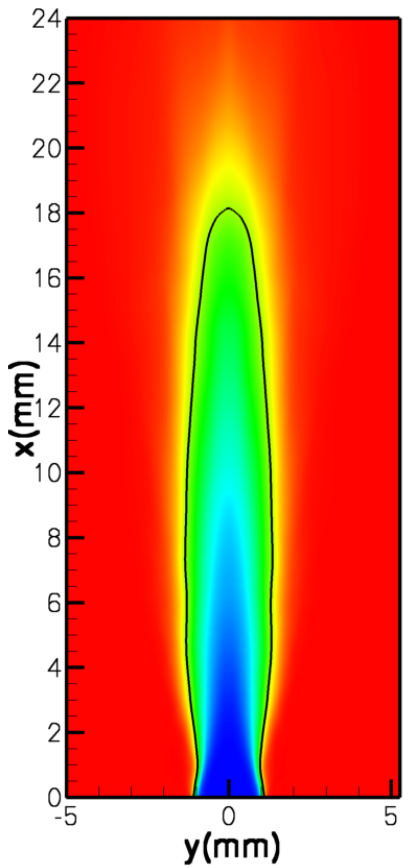

Case B

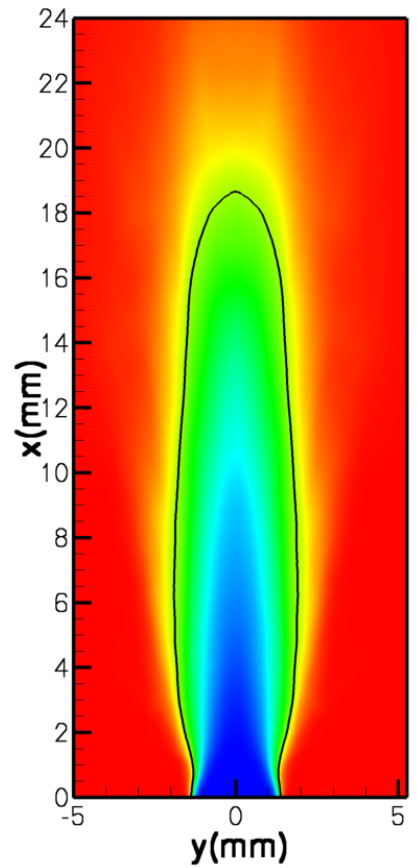

Case C

Figure 3: Favre averaged mean progress variable $(\tilde{c})$ is shown as a pseudocolor plot. The color scale varies from blue (0) to red (1). The iso-contour of $\tilde{c}=0.65$ is shown as a solid line. 

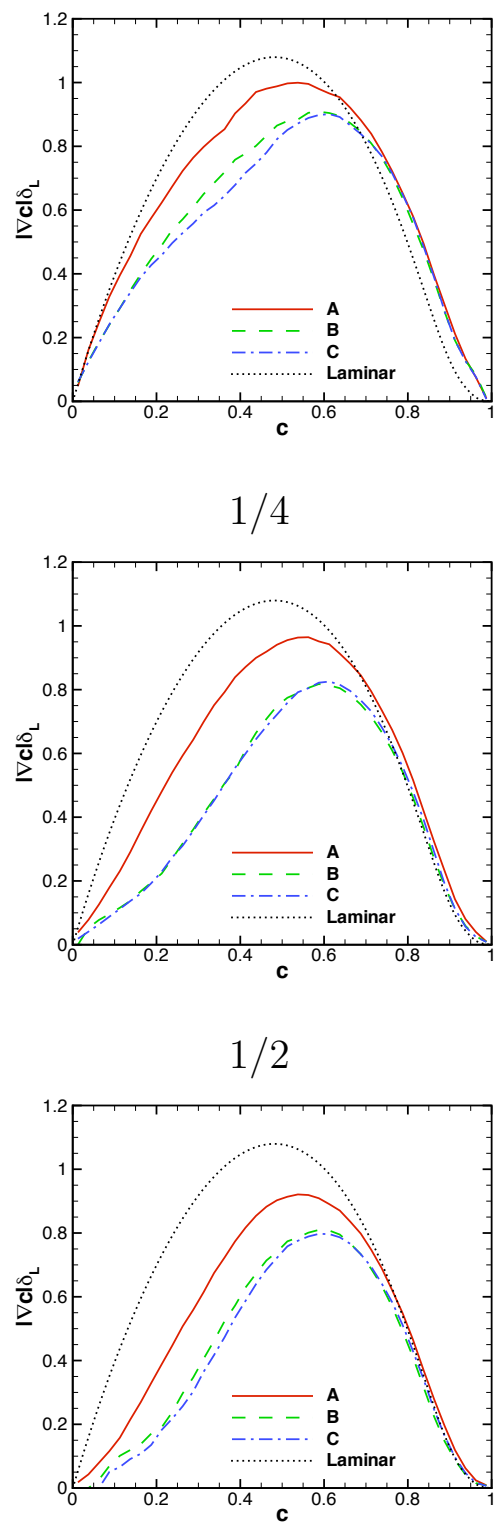

$3 / 4$

Figure 4: Conditional means of $|\nabla c|$ compared to the laminar flame profile for the three simulations. The means are computed at three chosen streamwise locations corresponding to $1 / 4,1 / 2$ and $3 / 4$ th of the domain length in the stream-wise direction. 


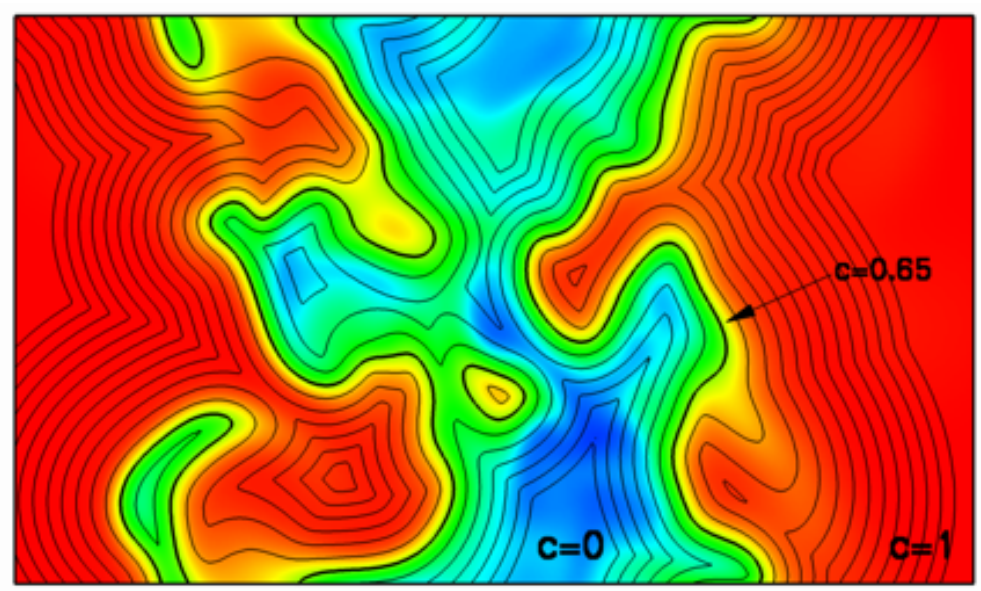

Figure 5: Instantaneous progress variable $(c)$ and distance function $(D)$ are shown in a slice of the domain perpendicular to the streamwise direction for case C. The color contours show $c$ on a blue $(c=0)$ to red $(c=1)$ scale. The lines represent iso-contours of $D$. The darkest iso-contour line represents $D=0$, which also corresponds to the iso-contour of $c=0.65$. 

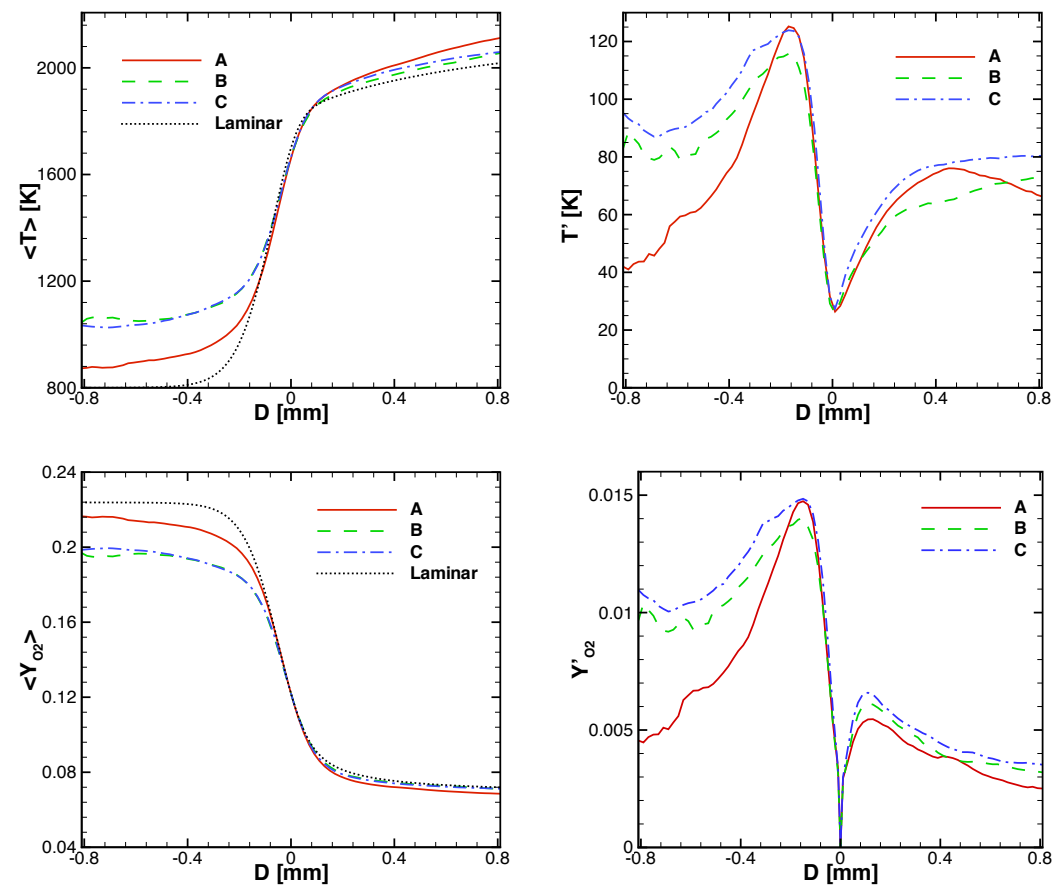

Figure 6: Mean and standard deviation of temperature and $Y_{\mathrm{O} 2}$ conditioned on the distance from the flame surface. Means are computed at the streamwise location ' $1 / 2$ '. 

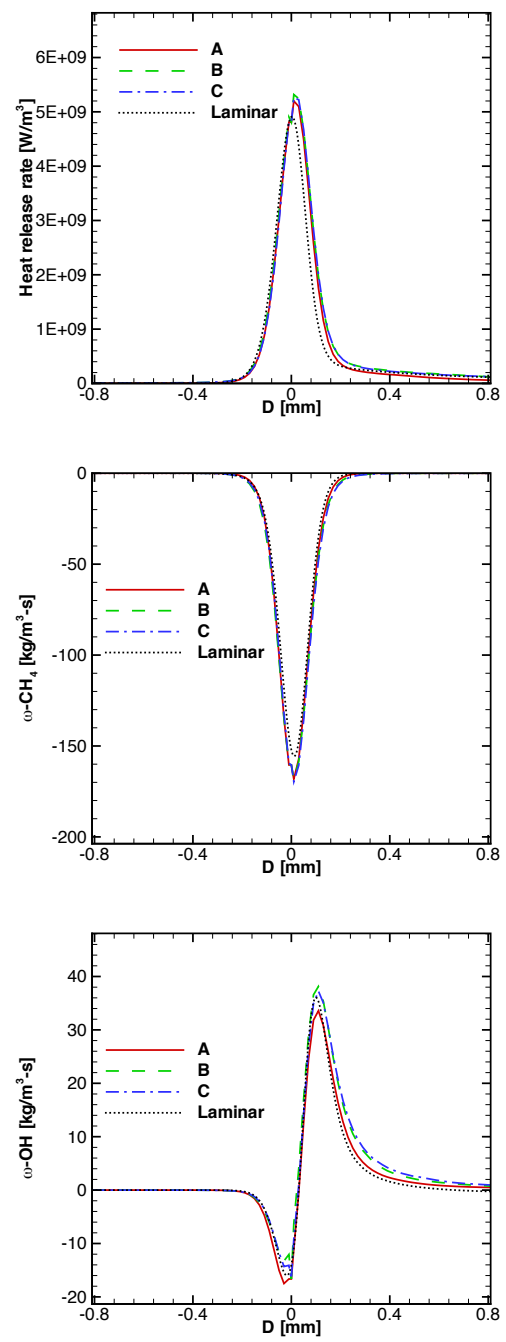

Figure 7: Mean heat release rate and reaction rates conditioned on the distance from the flame surface compared to the laminar profile. Results shown are at the $1 / 2$ streamwise location. The laminar flame results correspond to a strained laminar flame solution at strain rate $a_{\mathrm{t}} \tau_{\mathrm{f}}=1.2$ 


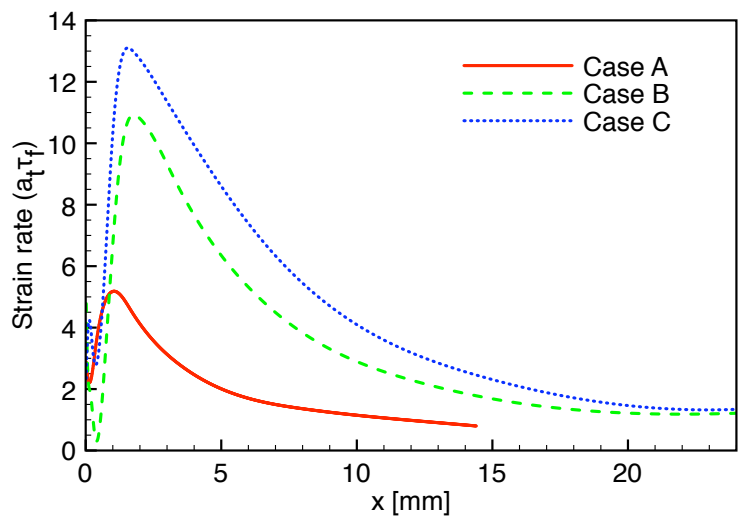

(a) $c=0.2$

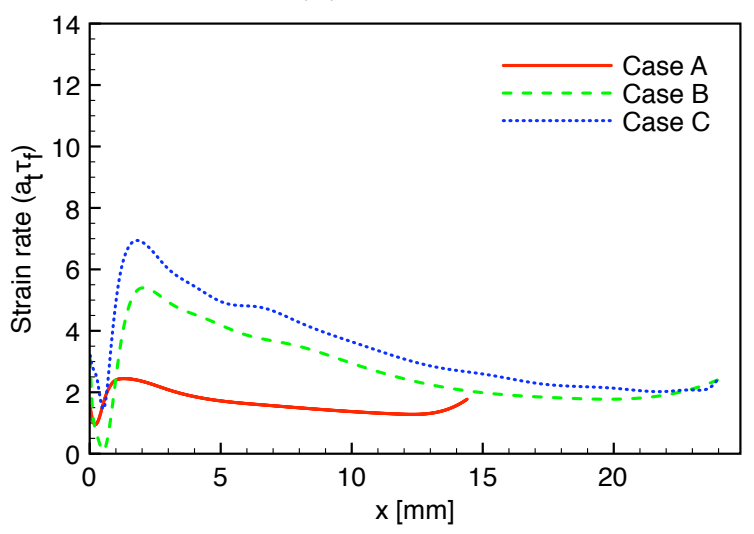

(b) $c=0.65$

Figure 8: Mean strain rate, conditioned on $c$, as a function of the axial distance. In (a) $c=0.2$ and in (b) $c=0.65$. 

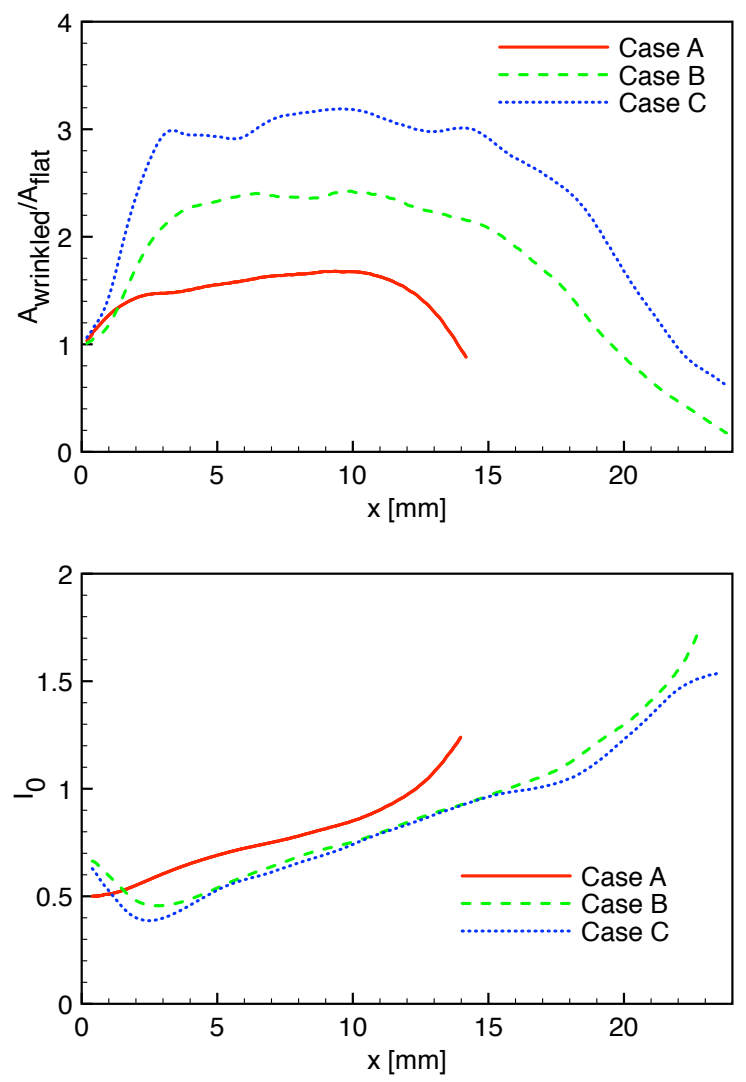

Figure 9: Flame area $\left(A^{\prime}\right)$ and burning efficiency $\left(I_{0}\right)$ variation along the axial direction. 


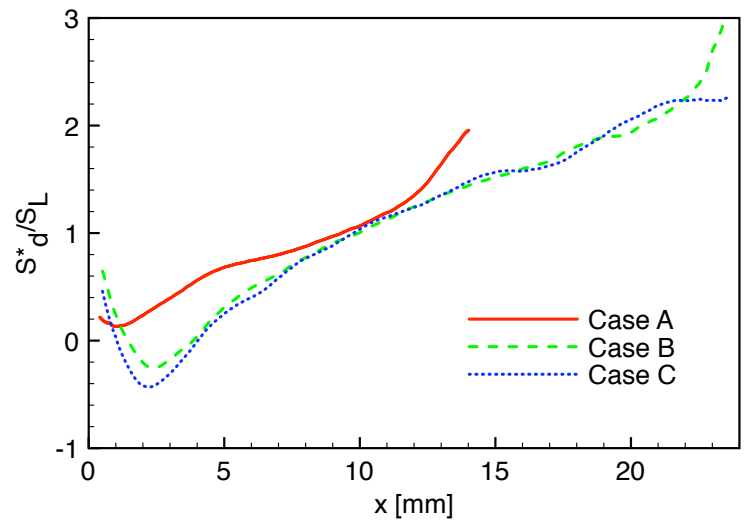

Figure 10: Mean $S_{\mathrm{d}}^{*}$, conditioned on $c=0.65$, as a function of the axial distance. 

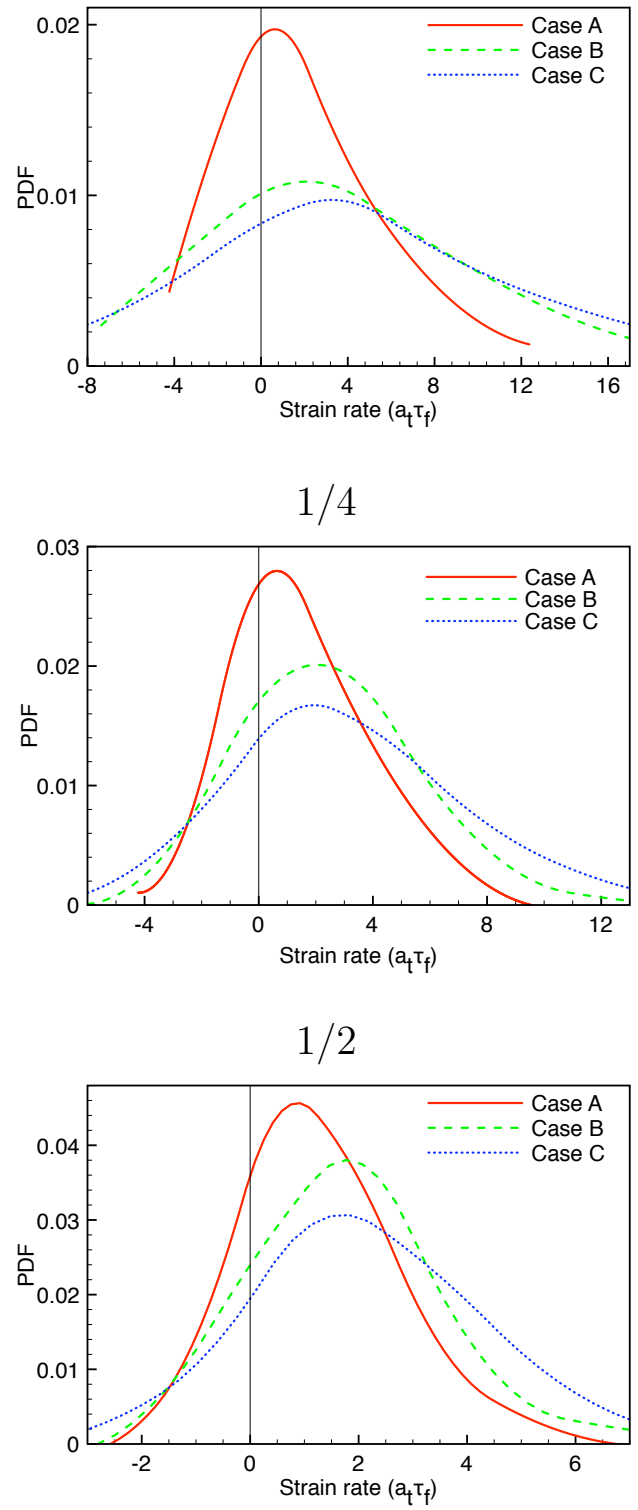

$3 / 4$

Figure 11: PDF of strain rate conditioned on $c=0.65$ at the $1 / 4,1 / 2$ and $3 / 4$ downstream locations. 

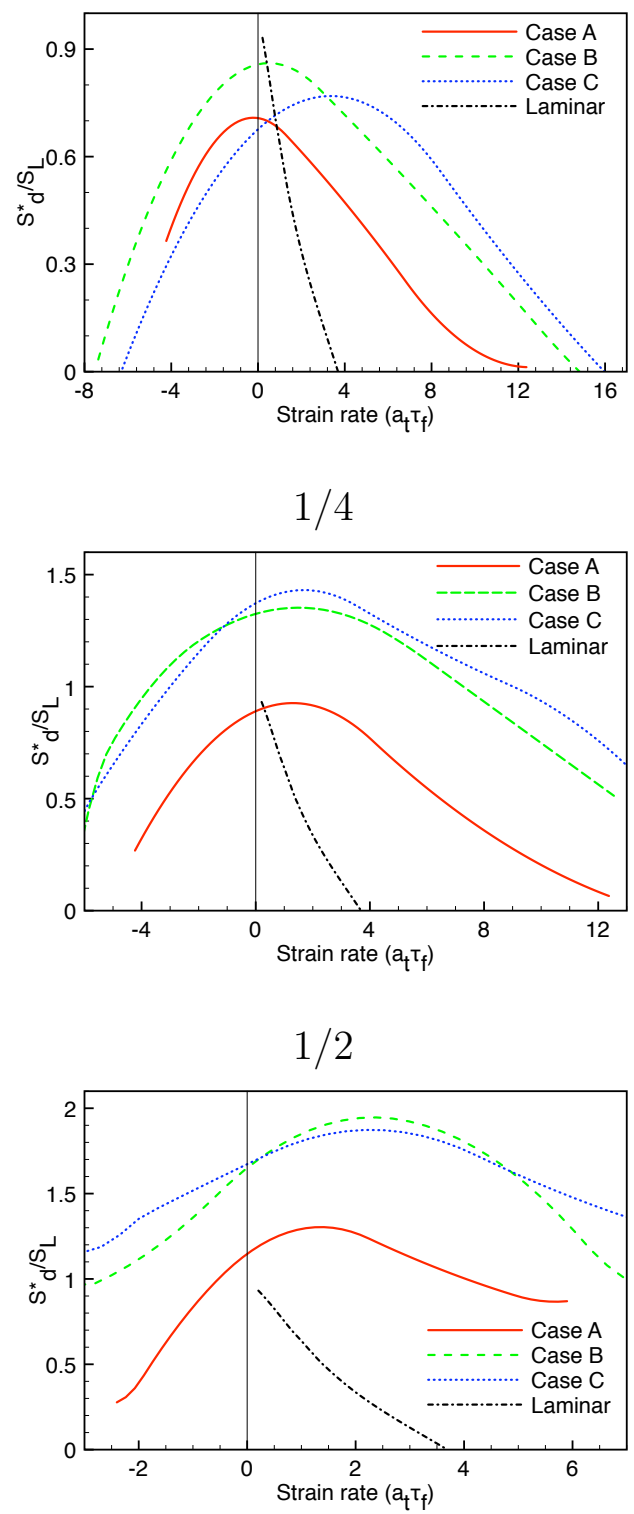

$3 / 4$

Figure 12: Strain-rate conditional PDF of the displacement speeds on the $c=0.65$ isosurface at $1 / 4,1 / 2$ and $3 / 4$ downstream locations. 


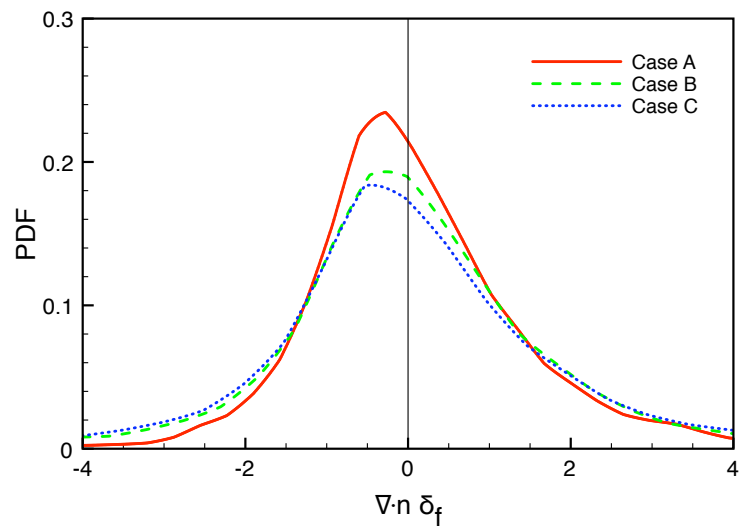

Figure 13: PDF of curvature conditioned on $\mathrm{c}=0.65$ at the $1 / 2$ downstream location. 


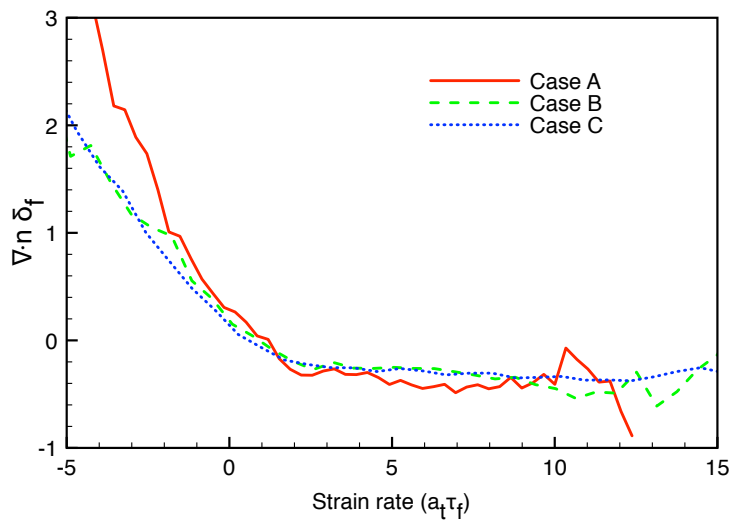

Figure 14: Strain-rate conditional mean of curvature on $c=0.65$ surface at the $1 / 2$ downstream location. 


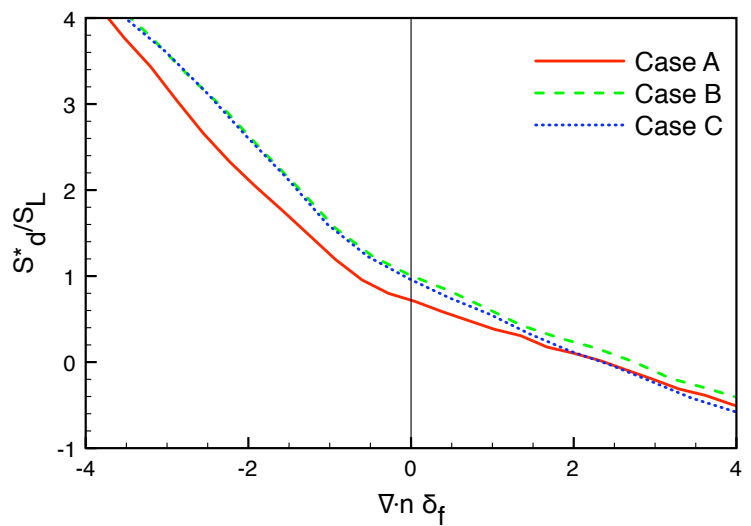

Figure 15: Curvature conditional mean $S_{\mathrm{d}}^{*}$ on c=0.65 at the $1 / 2$ downstream location. 


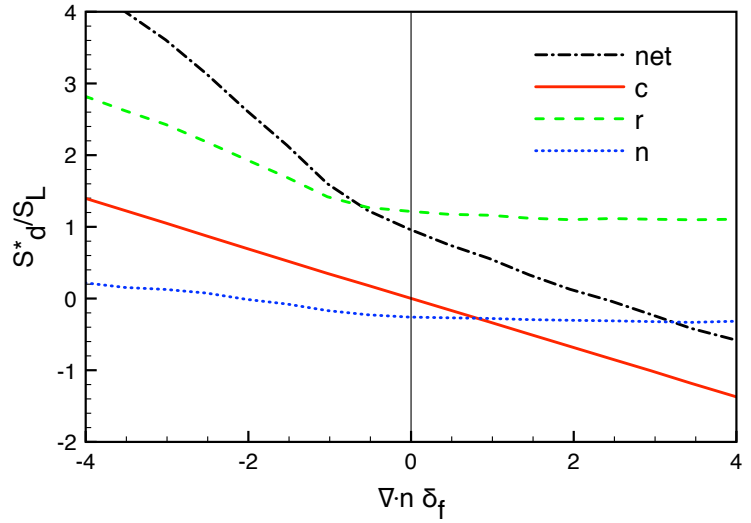

Figure 16: Curvature conditional mean $S_{\mathrm{d}}^{*}$ components on c=0.65 at the $1 / 2$ downstream location for case $\mathrm{C}$. 